\title{
A transferable coarse-grained model for diphenylalanine: How to represent an environment driven conformational transition
}

\author{
Cahit Dalgicdir, ${ }^{1}$ Ozge Sensoy, ${ }^{1, a)}$ Christine Peter, ${ }^{2,3}$ and Mehmet Sayar ${ }^{1, b}$ \\ ${ }^{1}$ College of Engineering, Koç University, 34450 Istanbul, Turkey \\ ${ }^{2}$ Max Planck Institute for Polymer Research, 55128 Mainz, Germany \\ ${ }^{3}$ Department of Chemistry, University of Konstanz, 78547 Konstanz, Germany
}

(Received 15 August 2013; accepted 27 November 2013; published online 20 December 2013)

\begin{abstract}
One of the major challenges in the development of coarse grained (CG) simulation models that aim at biomolecular structure formation processes is the correct representation of an environment-driven conformational change, for example, a folding/unfolding event upon interaction with an interface or upon aggregation. In the present study, we investigate this transferability challenge for a CG model using the example of diphenylalanine. This dipeptide displays a transition from a trans-like to a cis-like conformation upon aggregation as well as upon transfer from bulk water to the cyclohexane/water interface. Here, we show that one can construct a single CG model that can reproduce both the bulk and interface conformational behavior and the segregation between hydrophobic/hydrophilic medium. While the general strategy to obtain nonbonded interactions in the present CG model is to reproduce solvation free energies of small molecules representing the CG beads in the respective solvents, the success of the model strongly depends on nontrivial decisions one has to make to capture the delicate balance between the bonded and nonbonded interactions. In particular, we found that the peptide's conformational behavior is qualitatively affected by the cyclohexane/water interaction potential, an interaction that does not directly involve the peptide at all but merely influences the properties of the hydrophobic/hydrophilic interface. Furthermore, we show that a small modification to improve the structural/conformational properties of the CG model could dramatically alter the thermodynamic properties. ( 2013 AIP Publishing LLC. [http://dx.doi.org/10.1063/1.4848675]
\end{abstract}

\section{INTRODUCTION}

Peptides and proteins exhibit a strong tendency to form ordered aggregates. The amphiphilic nature of peptides plays an important role, by enabling aggregation in aqueous environment or at interfaces and surfaces or by allowing them to penetrate through or aggregate in membranes. ${ }^{1-3}$ Often the aggregation process can be controlled via stimuli that induce folding or conformational changes of the individual molecules which expose polar and apolar faces of the folded molecule. ${ }^{4}$ Or, vice versa, the aggregation of peptides or the interaction of a peptide with a hydrophobic/hydrophilic interface (or surface) triggers a conformational change in the molecule. Well known examples of the interplay of conformational change and aggregation or partitioning at interfaces are the "misfolding" of proteins upon amyloid aggregation, 5,6 or more generally the induction of higher $\beta$ sheet content by aggregation ${ }^{7}$ or by the presence of an interface. ${ }^{8,9}$ The above phenomena are of immense importance in a large variety of fields, such as biomedical research, food science, or material science. ${ }^{4,10-14}$ In all these cases, combinations of (peptide) folding, aggregation, interaction with interfaces, and partitioning between hydrophobic and hydrophilic media are essential elements that mutually influence each other.

\footnotetext{
a)Current address: Weill Cornell Medical College, New York, New York 10065, USA.

b)Electronic mail: msayar@ku.edu.tr
}

In order to better understand and ultimately control structure formation in peptide aggregates and peptide-based materials, knowledge of the relevant interactions, driving forces, pathways, and assembly mechanisms is essential. Here, molecular simulation can be a valuable tool to provide microscopic structural and thermodynamic insight into such systems, perfectly complementing experimental data and analytical models. However, since such structure formation processes occur on length and time scales that are frequently inaccessible to high-resolution all-atom models alone, coarse grained (CG) models are frequently employed - often hand in hand with more detailed atomistic simulations.

For the problems at hand it is essential that the interplay of aggregation, folding, and partitioning is correctly reflected in the CG model-in particular the correct representation of environment-driven conformational changes. This is a problem that can be viewed in the wider and more general context of transferability challenges: simulation models are state point dependent, i.e., they cannot per se be transferred to thermodynamic conditions, concentrations, chemical compositions, etc., that deviate from the original conditions where they had been parameterized. CG models are typically more state point dependent than higher resolution models, due to the reduction in the number of degrees of freedom where effective interactions are parameterized to capture the correct system behavior. Since understanding and solving transferability related limitations of CG models is crucial to their applicability to more complex (biomedical or materials science) systems, this 


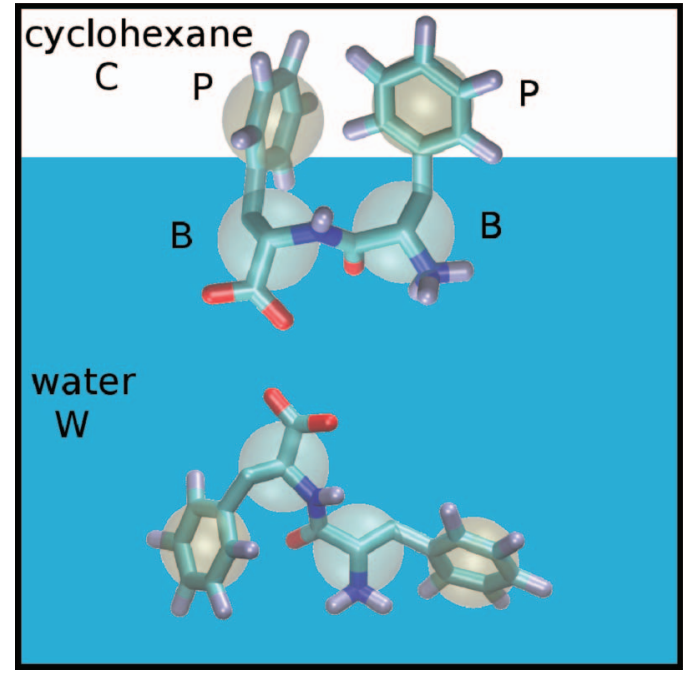

FIG. 1. Diphenylalanine peptide in cis-like conformation at the cyclohexane/water interface (top) and in trans-like conformation in bulk water (bottom). Mapping of the peptide to a 4 bead CG model (PBBP, $\mathbf{P}$ for sidechain and $\mathbf{B}$ for backbone beads) is also shown. Solvent molecules (shown as continuum for visual clarity) are mapped onto single beads: $\mathbf{W}$ and $\mathbf{C}$ for water and cyclohexane, respectively.

topic has emerged as a high priority area in the development of coarse graining methods.

In this study, we develop a transferable CG model for a peptide which is capable of reproducing the correct conformational behavior in two different environments. The zwitterionic diphenylalanine peptide (FF) displays a conformational transition from a trans-like to a cis-like state upon formation of a crystal structure composed of a hydrophobic matrix (formed by the sidechains) with hydrophilic water channels (surrounded by the FF backbone and charged end-groups). ${ }^{15}$ This rather unusual backbone conformation is stabilized by the hydrophobic medium resulting from the neighboring FF molecules' sidechains. Here, we show via atomistic simulations that the FF molecule shows a similar conformational transition when it is brought in contact with a hydrophobic/hydrophilic interface (see Figure 1). We aim to build a CG model for FF, which can successfully reproduce the conformational behavior of the molecule in both bulk water and cyclohexane/water interface. This behavior as well as its small size and remarkable self-assembly capacity ${ }^{16}$ make FF an ideal model system for a systematic study of rather fundamental questions regarding the interplay of nonbonded interactions and conformational behavior, specifically in the context of structure- vs. thermodynamics-based coarse graining methodologies.

\section{METHODS}

Atomistic MD simulations were performed for a single FF molecule in bulk water and in a cyclohexane/water biphasic system representing the behavior at a hydrophobic/hydrophilic interface by using GROMACS 4.5 simulation package. ${ }^{17}$ The conformational distributions of the atomistic simulations of FF in both environments are used as the references for devising the CG model. For the bulk water simulation a cubic box with a dimension of $5 \mathrm{~nm}$ is used and it contains 4123 water molecules. The cyclohexane/water biphasic system for the interface simulations is performed in a box of size $4 \times 4 \times 6.5 \mathrm{~nm}$ and contains 1601 water and 267 cyclohexane molecules. Box sizes are chosen to be larger than twice the largest cut-off value to prevent interactions with periodic images.

Atomistic simulations were obtained by using the leapfrog integrator with a time-step of 2 fs. GROMOS $53 \mathrm{a} 6$ force-field ${ }^{18}$ is used for the FF molecule and SPC/E model $^{19}$ for water. Hydrogen bonds were constrained with Lincs algorithm. ${ }^{20}$ Temperature is set to $298 \mathrm{~K}$ via velocityrescaling algorithm ${ }^{21}$ with a coupling time constant of 1 ps. For bulk water simulations isotropic Berendsen pressure coupling at a reference pressure of $1 \mathrm{~atm}$ with a coupling time constant of $1 \mathrm{ps}$ and a compressibility of $4.5 \times 10^{-5} \mathrm{bar}^{-1}$ is used. For the cyclohexane/water interface simulations semiisotropic pressure coupling is used such that the pressure along the $\mathrm{z}$ direction is maintained at $1 \mathrm{~atm}$ and dimensions of the box in $\mathrm{x}$ and $\mathrm{y}$ directions are held constant. Electrostatic interactions are handled by particle mesh Ewald (PME) ${ }^{22}$ with a Coulomb cutoff of $1 \mathrm{~nm}$.

For the coarse-grained simulations the atomistic reference systems were mapped onto the CG model representations of the molecules by using VOTCA package, ${ }^{23}$ while maintaining the same number of molecules. CG simulations were performed in canonical ensemble (NVT) to avoid large volume fluctuations. The volume of the CG box was reduced to match the reference pressure of $1 \mathrm{~atm}$ : for bulk water simulations a cubic box of length $4.74 \mathrm{~nm}$ and for the interface simulations a rectangular box of size $4 \times 4 \times 6 \mathrm{~nm}$. Van der Waals (VDW) interactions were cut off at $1.4 \mathrm{~nm}$. For the CG simulations a larger timestep ( $5 \mathrm{fs}$ ) was used, which accelerates the CG simulations by a factor of 2.5 , while maintaining correct sampling for the stiffest bond in our CG model. All remaining simulation parameters were kept identical to the atomistic case.

Surface tensions of the atomistic and CG interface systems were calculated from the difference between the normal and the lateral pressure as described in detail in the Gromacs manual, ${ }^{24}$ Eqs. (3.61) and (3.62). All molecular visualizations were performed by Visual Molecular Dynamics (VMD) package. ${ }^{25}$

Block analysis method was used to check the convergence of the probability distributions for all systems. Analysis results for atomistic and CG interface systems are given in Figs. S1 and S2 of the supplementary material. ${ }^{26}$

Free energy calculations were performed by thermodynamic integration using Bennett acceptance ratio (BAR) method. ${ }^{27,28}$ The free energy calculation simulations are performed under isothermal-isobaric ensembles (NPT) with stochastic dynamic integrator, ${ }^{29}$ using Berendsen pressure coupling. ${ }^{30}$

Umbrella sampling ${ }^{31}$ was used for potentials of mean force (PMF) calculations where a harmonic constraint potential was applied between the centers of mass of the water slab and the FF dipeptide. Cosine weighting ${ }^{32}$ was applied for the center of mass of the water slab. FF dipeptide is pulled along the direction perpendicular to the interface (z-direction) 
with a pull rate of $0.01 \mathrm{~nm} / \mathrm{ps}$ with a harmonic constant of $1000 \mathrm{~kJ} \mathrm{~mol}^{-1} \mathrm{~nm}^{-2}$ and snapshots of the system are taken at regular intervals. Energy minimization using the steepestdescent algorithm and an NVT equilibration for 50000 steps were subsequently applied to each of these snapshots which were then used as the initial configurations for the umbrella sampling windows. Each constraint distance for the fullatomistic and CG simulations was sampled for $10 \mathrm{~ns}$ and $50 \mathrm{~ns}$, respectively. Weighted histogram analysis method ${ }^{33}$ was used to obtain the PMF curves.

\section{TRANSFERABILITY OF CG MODELS: DIFFERENT CHEMICAL ENVIRONMENTS}

A common approach in development of coarse-grained models is to adopt a Hamiltonian with separate terms for bonded and nonbonded interactions:

$$
U^{c g}=U_{\text {bonded }}^{c g}+U_{\text {nonboned }}^{c g},
$$

where $U^{c g}$ is the total coarse-grained potential. Similarly, bond-length, angle-bending, and dihedral interactions are included into the Hamiltonian as separate terms,

$$
U_{\text {bonded }}^{c g}=U_{r}^{c g}+U_{\theta}^{c g}+U_{\phi}^{c g} .
$$

Often then the parametrization of the different terms is done independently, implying that the relevant degrees of freedom are decoupled, or even if the resulting bonded and nonbonded distributions are weakly correlated, the coarse-grained model will successfully mimic the all atom case and display an identical or similar coupling. Hence, the strategy to devise nonbonded interactions plays no role for parametrization of the bonded potentials. A discussion on these assumptions, where they fail, and how the problems can be overcome has already been provided elsewhere. ${ }^{34,35}$

Conformational probability distributions, obtained by mapping the atomistic configurations to the respective coarse grained coordinates, can be used to obtain PMF for each interaction, ${ }^{36-38}$

$$
\begin{gathered}
U^{c g}(r, T)=-k_{B} T \ln \left(P^{c g}(r, T) / r^{2}\right)+C_{r}, \\
U^{c g}(\theta, T)=-k_{B} T \ln \left(P^{c g}(\theta, T) / \sin (\theta)\right)+C_{\theta}, \\
U^{c g}(\phi, T)=-k_{B} T \ln \left(P^{c g}(\phi, T)\right)+C_{\phi},
\end{gathered}
$$

where $P^{c g}(r, T)$ is the bonded, $P^{c g}(\theta, T)$ is the angle-bending, and $P^{c g}(\phi, T)$ is the dihedral distribution. $k_{B}$ is the Boltzmann constant, $T$ is the temperature, and $C$ 's are constant values.

Available methods for parametrization of nonbonded interaction potentials can be divided into two general categories: (i) methods where the CG parameters are refined so that the system displays a certain thermodynamic behavior (typically termed thermodynamics-based) ${ }^{39-43}$ or (ii) methods where the CG system aims at reproducing the configurational phase space sampled by an atomistic reference system (often misleadingly termed structure-based). ${ }^{36,44-55}$ Inherent to the process of coarse graining, the resulting CG models typically suffer from representability problems: ${ }^{56}$ i.e., a structure-based approach does not necessarily yield correct thermodynamic properties such as solvation free energies or partitioning data while thermodynamics-based potentials may not reproduce microscopic structural data such as the local packing or the structure of solvation shells. Thus, a careful assessment of the system and problem of interest needs to be made before choosing an appropriate coarse graining strategy.

Closely related problems arise from the limited transferability of CG models: all CG models (in fact, also all classical atomistic forcefields) are state-point dependent and cannot necessarily be-without reparametrization-transferred to different thermodynamic conditions (temperature, density, concentration, system composition, phase, etc.) or a different chemical or molecular environment (e.g., a certain chemical unit being part of different macromolecular chains). Structural and thermodynamic representability and state-point transferability questions are often intimately linked, since the response to a change in state point corresponds to representing certain thermodynamic properties, and intensive research is currently devoted to this problem. ${ }^{51,52,57-63}$

To study biomolecular processes such as peptide aggregation and biomaterials structure formation, CG models are often developed based on smaller less complex reference systems (individual peptides, subsystems, etc.). Consequently, it is essential to achieve transferability among different concentrations and environments, to maintain correct partitioning and solvation properties, and to reproduce coupling between conformational behavior and environment.

In the present study, we have entirely focused on the interplay of bonded and nonbonded interaction functions, in particular upon the transition from a bulk water environment to a hydrophilic/hydrophobic (cyclohexane/water) interface. We will use the FF molecule as a model system, and demonstrate how one can parametrize a CG model which is capable of representing the conformational properties of the molecule in two different environments.

In its crystal structure, FF adopts a cis-like conformation, where the two phenyl rings point in the same direction. ${ }^{15}$ In the following, we will show that a single FF molecule in water displays a trans-like conformation, and that the corresponding structural change can be triggered by exposure to a hydrophobic interface or aggregation. As such, the FF molecule is an ideal model system for our study, and in order to understand and characterize the conformational preference of the FF molecule we will first present the results of three different atomistic simulations.

When a single FF molecule is simulated in bulk water, the molecule adopts a trans-like conformation as shown in Fig. 1 (bottom). Such a conformation relaxes the backbone dihedral angles, but the hydrophobic phenylalanine side chains are separated from each other and fully hydrated. The different conformations of FF can best be characterized via a dihedral angle between four superatoms (also indicated in Fig. 1). Note that, later these superatoms are also going to be used as the interaction sites in the CG model, i.e., they reflect the mapping scheme that relates the $\mathrm{CG}$ beads with the atomistic coordinates of FF. Two superatoms which are positioned in the respective centers of mass of the phenylalanine rings will be referred to as $\mathbf{P}$. The backbone and charged 


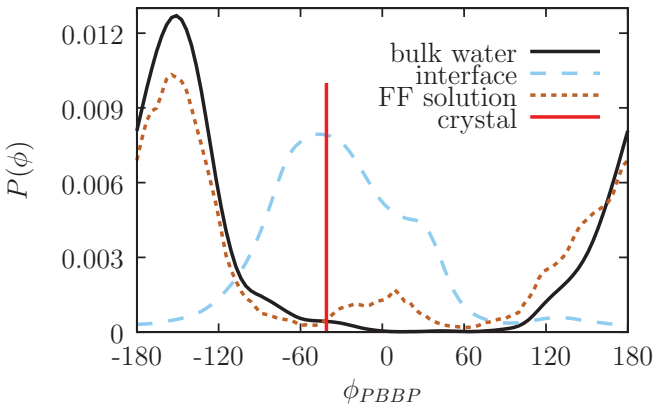

FIG. 2. Probability distribution for the PBBP dihedral angle from atomistic simulations. In bulk water (solid black line), the molecule adopts an extended structure. At the cyclohexane/water interface the molecule adopts a cis-like conformation (dashed blue line), highly distinct from bulk water behavior, but similar to the crystal structure (solid red impulse). In a finite-concentration solution of FF peptide molecules can switch from a trans-like to a cis-like conformation (probability distribution for such a molecule is shown with dashed brown line), when presented with a hydrophobic interface formed by a small cluster of FF molecules.

end-groups of the FF molecule will be represented by two identical superatoms, which are located at the positions of the two $\mathrm{C}_{\alpha}$ atoms and which will be referred to as $\mathbf{B}$. The probability distribution for the dihedral angle among PBBP superatoms in bulk water is peaked at an extended conformation with a maximum around $-150^{\circ}$ (Fig. 2 , solid black line).

In atomistic simulations of a biphasic cyclohexane/water environment, the FF molecule (initially, placed in the water layer) quickly adsorbs to the interface, and adopts a cis-like conformation as indicated in the representative snapshot in Fig. 1 (top). The dihedral probability distribution at the interface (Fig. 2, dashed blue line) is very distinct from the bulk water distribution and its maximum matches perfectly with the dihedral adopted in the crystal structure ${ }^{15}$ (shown with the red impulse line in Fig. 2). Phenyl side chains are driven into the cyclohexane phase via hydrophobic forces and this force triggers an environment mediated conformational transition in the molecule.

The conformational transition that is observed at the interface can also take place in bulk water with a finite concentration of FF molecules as observed in our simulations and previously by Jeonet al. ${ }^{64}$ As the FF molecules start forming an aggregate, the hydrophobic environment, provided by the surrounding phenylalanine side chains, lowers the energy barrier for the transition to a cis-like conformation. As a result, a certain fraction of the FF molecules sample the cis-like conformation during the simulation. A representative snapshot for the conformation of such a molecule is shown in Fig. 3. The yellow colored molecule displays a cis-like conformation when its sidechains are in contact with a hydrophobic/hydrophilic interface of a nano-cluster of neighboring molecules. The dihedral distribution of the corresponding molecule clearly shows (Fig. 2, dashed brown line) two peaks, one corresponding to the trans-like (characteristic for bulk water environment) and one to the cis-like (characteristic for the interface behavior) conformation. This behavior is not specific to a single molecule, but can be seen for many such molecules (see Fig. S3 of the supplementary material ${ }^{26}$ ). Note that this conformational transition is only temporary as

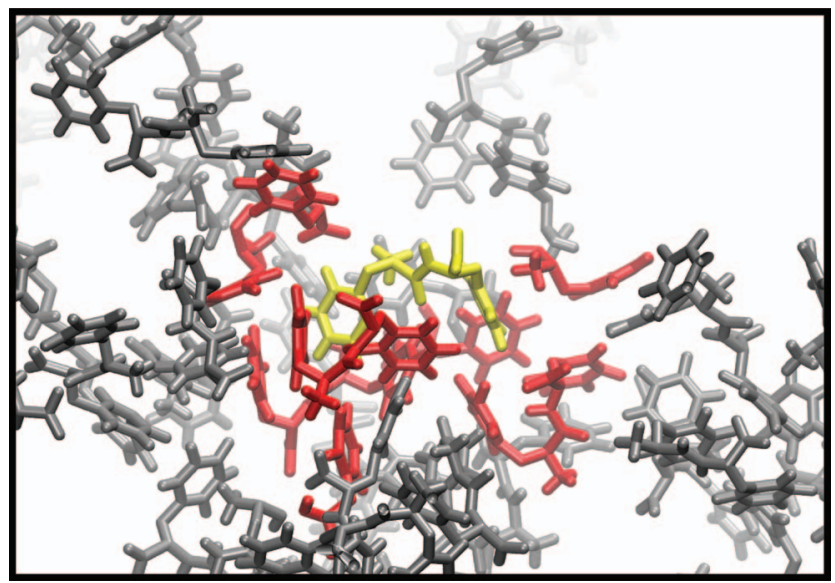

FIG. 3. A diphenylalanine in cis-like conformation (shown in yellow) at the interface of a nano-cluster of diphenylalanine molecules in water. Water molecules are not shown for clarity. The nano-cluster of FF molecules presents a hydrophilic/hydrophobic interface, which enables the transition of the corresponding molecule to a cis-like conformation. Peptide residues within $4 \AA$ of the corresponding peptide are shown in red color.

seen in the time line evolution of the dihedral angle (Fig. 4). During the $200 \mathrm{~ns}$ simulation, FF molecules form small clusters, however, no stable nucleus for crystallization is observed.

Despite the dramatic change in the dihedral angle, the bond length and angle distributions show only modest changes upon transfer of the molecule from bulk water to the interface. For each bond and angle distribution the bulk and interface results are shown in Figures 5 and 6, with solid and dashed lines, respectively. Except for a small shift in the BB bond length, in general bond lengths remain identical in bulk water and interface simulations. For the bond angle distributions, a bimodal distribution is observed for both BBP and PBB angles. Even though one can see a slight shift in peak location and change in height, compared to the dihedral angle these can be considered as secondary minor changes.

This small molecule represents an interesting challenge for coarse-graining methodology. In order to design a coarsegrained model which can capture the behavior of the molecule both in bulk water and at the cyclohexane/water interface, one has to reconsider the assumption of the decoupling of bonded

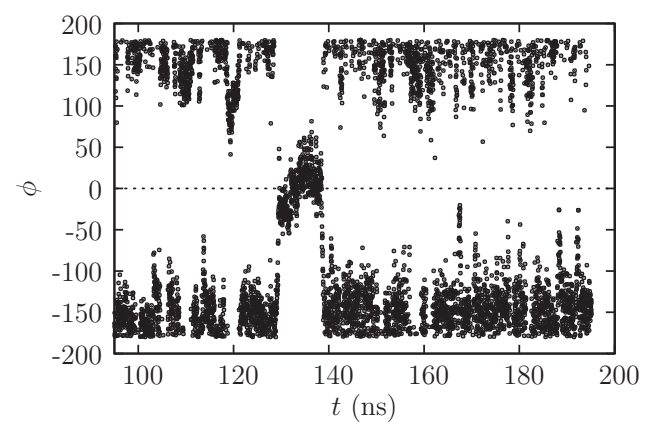

FIG. 4. Dihedral angle among PBBP superatoms of the molecule in Fig. 3 as a function of simulation time. Unlike the peptide in bulk water (where only trans-like conformation is observed), in a solution of peptides in water, formation of nano-clusters with hydrophilic/hydrophobic interfaces enables sampling of the cis-like conformation. 


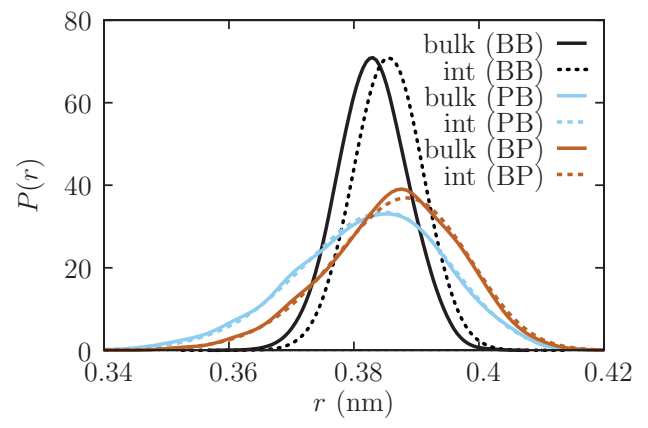

FIG. 5. Probability distributions for bonds between FF superatoms (BB, $\mathrm{PB}$, and $\mathrm{BP}$ ) from atomistic simulations in bulk water and at the cyclohexane/water interface.

and nonbonded interactions-where the latter encompass solvent-solvent as well as solute-solvent interactions. The dihedral angle PBBP displays an environment driven transition, from a trans-like conformation in bulk water to a cis-like conformation at the interface. It cannot be taken for granted that the combination of a dihedral potential based on the bulk dihedral angle distribution (which strongly favors the trans state) with the CG nonbonded interactions of $\mathrm{P}$ and $\mathrm{B}$ beads with the solvent molecules will result in an identical change of conformation when the molecule is placed at the interface. One would rather expect that the transition behavior should be strongly dependent on the type of parametrization of the nonbonded CG model, in particular one would suspect the nonbonded interaction of $\mathrm{P}$ and $\mathrm{B}$ beads with the solvent molecules to be relevant. In Sec. IV, we present our approach to developing a transferable coarse-grained model for FF, and demonstrate its success and drawbacks.

\section{TRANSFERABLE CG MODEL DEVELOPMENT AND RESULTS}

\section{A. CG solvent model}

As we aim to analyze the conformation of the FF molecule both in bulk water and at the cyclohexane/water interface, we used an explicit solvent CG model. Both the water and cyclohexane solvent molecules are mapped onto one bead per molecule ( $\mathbf{W}$ for water and $\mathbf{C}$ for cyclohexane), which is positioned at the center of mass of the corresponding solvent

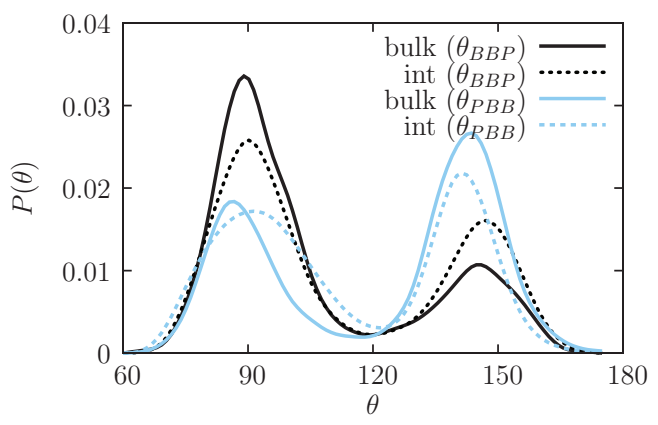

FIG. 6. Probability distributions between the BBP and PBB superatom triplets from atomistic simulations in bulk water and at the cyclohexane/water interface.
TABLE I. Solvation free energies of water and cyclohexane molecules for the constituent subphases of the interface system measured from atomistic (AA) simulations. van der Waals and electrostatic contributions, as well as the total free energy change are listed. Lennard-Jones interactions, where parameters $\epsilon$ and $\sigma$ are tuned to reproduce the measured atomistic free energy, are used for the nonbonded interactions of water $(\mathbf{W})$ and cyclohexane $(\mathbf{C})$ CG beads. Parametrization of the $\mathbf{W}-\mathbf{C}$ interaction is done in two different ways: (1) CG-FE: solvation free energy of water in cyclohexane (W/C) is used as reference. (2) CG-ST: interaction is tuned to reproduce the surface tension of the biphasic system.

\begin{tabular}{lcccccc}
\hline \hline Ref./Sol. & System & $\begin{array}{c}\text { VdW } \\
(\mathrm{kJ} / \mathrm{mol})\end{array}$ & $\begin{array}{c}\text { Elec. } \\
(\mathrm{kJ} / \mathrm{mol})\end{array}$ & $\begin{array}{c}\text { Total } \\
(\mathrm{kJ} / \mathrm{mol})\end{array}$ & $\begin{array}{c}\epsilon \\
(\mathrm{kJ} / \mathrm{mol})\end{array}$ & $\begin{array}{c}\sigma \\
(\mathrm{nm})\end{array}$ \\
\hline W/W & AA & 9.02 & -39.24 & -30.22 & - & - \\
& CG & -28.36 & - & -28.36 & 4.64 & 0.288 \\
C/C & AA & -17.60 & - & -17.60 & - & - \\
& CG & -16.95 & - & -16.95 & 4.0 & 0.547 \\
W/C & AA & 2.86 & - & 2.86 & - & - \\
& CG-FE & 2.71 & - & 2.71 & 1.33 & 0.455 \\
& CG-ST & -10.62 & - & -10.62 & 3.19 & 0.455 \\
C/W & AA & 9.17 & - & 9.17 & - & - \\
& CG-FE & 114.4 & - & 114.4 & 1.33 & 0.455 \\
& CG-ST & 41.30 & - & 41.30 & 3.19 & 0.455 \\
\hline \hline
\end{tabular}

molecule. For the parametrization of the nonbonded solventsolvent interactions a Lennard-Jones 9-6 potential is used. The radii $(\sigma)$ for the Lennard-Jones interaction are matched to the first peak in the radial distribution functions in the AA simulations. The depth of the Lennard-Jones interaction $(\epsilon)$ is tuned to reproduce AA solvation free energies of the respective groups, which are measured via thermodynamic integration. ${ }^{65}$ All free energy values (both from AA and CG simulations) that are discussed in the following, as well as the $\epsilon$ and $\sigma$ values for all solvent parameters are listed in Table I. The respective potentials are also graphically depicted in Fig. 7.

The pure solvent (i.e., $\mathbf{W}-\mathbf{W}$ and $\mathbf{C}-\mathbf{C}$ ) interactions are parametrized to match the AA thermodynamic integration results from the self-solvation of the corresponding molecules. For the parametrization of the (mixed) W-C interaction the case is a little more ambiguous: in principle one has two reference values from the solvation of cyclohexane in water $\mathbf{C} / \mathbf{W}$

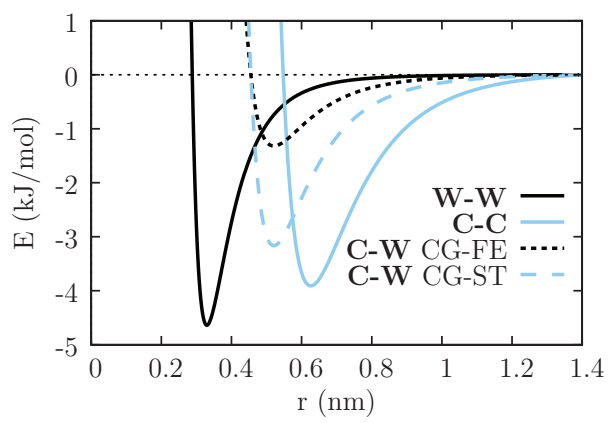

FIG. 7. CG non-bonded interaction potentials for the solvent beads (Lennard-Jones parameters are given in Table I). W-W and C-C interactions are shown with solid black and blue lines, respectively. Two different potentials are used for the W-C interaction, where either the solvation free energy of water in cyclohexane (CG-FE, dashed black line) or the surface tension of cyclohexane/water system (CG-ST, long-dashed blue line) is taken as a reference. 
and from the solvation of water in cyclohexane $\mathbf{W} / \mathbf{C}$, and ideally the mixed interaction should reproduce both values.

As a first approach we tuned the CG W-C interaction to reproduce the AA solvation free energy of water in cyclohexane (W/C). The CG free energy changes linearly with the depth of the potential $\epsilon$, and after a few iterations we set $\epsilon=1.33 \mathrm{~kJ} / \mathrm{mol}$, which yields the CG solvation free energy of W/C to $2.71 \mathrm{~kJ} / \mathrm{mol}$-fairly close to the AA result $(2.86 \mathrm{~kJ} / \mathrm{mol})$. Using this interaction function, the CG solvation free energy of cyclohexane in water $(\mathbf{C} / \mathbf{W})$ is measured as $114.4 \mathrm{~kJ} / \mathrm{mol}$, which is an overestimation compared to the AA result $(9.17 \mathrm{~kJ} / \mathrm{mol})$, i.e., cyclohexane beads are too strongly repelled from the aqueous phase. If, alternatively, one chooses to tune the CG interaction potential for $\mathbf{W}-\mathbf{C}$ to match the $\mathbf{C} / \mathbf{W}$ AA solvation free energy, one obtains a very deep W-C interaction (data not shown). This model results in a negative W/C solvation free energy, i.e., the solvation of water in cyclohexane becomes highly favorable, destabilizing the interface. Hence, this model clearly leads to unphysical results. It turns out that with the approach chosen one cannot simultaneously reproduce both the W/C and the $\mathbf{C} / \mathbf{W}$ solvation free energy in the CG simulations. The first CG model-tuned based on the W/C solvation free energy-will be referred to as CG-FE solvent model in the remainder of the text.

Given the ambiguity discussed above we decided to alternatively tune the $\mathbf{W}-\mathbf{C}$ interaction potential to reproduce the surface tension of the cyclohexane/water system (with the target surface tension from AA simulations being $50.2 \mathrm{mN} / \mathrm{m}$ ). The resulting model will be referred to as CG-ST solvent model (see Fig. 7, the potential is significantly deeper than CG-FE but not as deep as the discarded free-energy model tuned from the $\mathbf{C} / \mathbf{W}$ solvation). Compared to the CG-FE solvent model, which yields a surface tension of $157 \mathrm{mN} / \mathrm{m}$, CG-ST by construction better represents the interface properties. However, this is achieved at the cost of the solvation free energies in the corresponding subphases of the system: the solvation free energy for $\mathbf{W} / \mathbf{C}$ and $\mathbf{C} / \mathbf{W}$ are calculated as $-10.62 \mathrm{~kJ} / \mathrm{mol}$ and $41.30 \mathrm{~kJ} / \mathrm{mol}$, respectively (see Table I). As a further comparison of the CG solvent models with AA data, radial distribution functions and density profiles for water and cyclohexane beads are shown in Figs. S4 and S5 of the supplementary material. ${ }^{26}$

For the parametrization of $\mathrm{FF}$ in the interface system, in Sec. IV B we will proceed with both CG solvent models, CGFE and CG-ST. Note though that the only difference between the models is the interaction between the CG cyclohexane and water beads, i.e., the interaction that governs the behavior of the interface.

\section{B. CG FF model}

The CG model for FF used here is composed of four beads as already described and depicted in Fig. 1, with masses of the sidechains assigned to the $\mathbf{P}$ beads and the mass of the backbone atoms and the end groups distributed equally to the two $\mathbf{B}$ beads. As discussed above, the environment-driven conformational transition of FF can be successfully represented in this mapping scheme via the PBBP dihedral angle.
Since the solvent superatoms are not polarizable and in the present simulations no peptide-peptide interactions are considered, the $\mathbf{B}$ beads on the peptides do not carry any charge.

With this mapping the CG FF model requires 3 bond, 2 angle, and 1 dihedral angle as bonded interactions, and nonbonded interaction potentials between the peptide and the solvent molecules. As a further simplification, we ignored the small differences in bond and angle distributions between bulk water and at the interface, and focus primarily on the dihedral angle. The interaction potentials for bonds (PB, BP, BB) and angles (PBB, BPP) are obtained via Boltzmann inversion (Eqs. (3)-(5)) of the corresponding probability distributions in bulk water (see Figs. 5 and 6). The bonds are represented via harmonic potentials (see Table S1 of the supplementary material ${ }^{26}$ ), whereas the angle and dihedral potentials are represented in tabular form (see Figs. S6 and S7 of the supplementary material ${ }^{26}$ ). The resulting distributions from the CG model and comparison with the atomistic bulk and interface simulations are given in Figs. S8 and S9 of the supplementary material. ${ }^{26}$ For the bond and angle distributions the CG model reproduces the AA system well. Hence, we only focused on the dihedral angle PBBP.

Since the PBBP dihedral angle is coupled to the nonbonded interactions, we first describe the initial parametrization of the nonbonded interactions between the peptide and the solvent molecules. Similar to the solvent parametrization, these are also derived based on the solvation free energy of fragments representing the respective groups obtained via thermodynamic integration. Note that the derivation of parameters based on molecule fragments is potentially problematic since free energy is not per se additive, but these fragment-based parameters serve as a good initial guess. The free energy change upon transfer of the relevant group from vacuum into the bulk solvent medium is obtained from AA simulations. The relevant solvation free energy values measured from AA simulations, and the Lennard-Jones parameters for the matched CG interaction and the resulting CG free energy values are shown in Table II. Ethyl-benzene

TABLE II. Solvation free energy of FF fragments (that are mapped to the CG beads) in the respective solvents obtained from AA simulations. CG beads' nonbonded interactions are represented via Lennard Jones interactions, where the depth $\epsilon$ and radius $\sigma$ of the interaction are fitted to reproduce the AA free energy. Results with the modified $\mathbf{P} / \mathbf{C}$ interaction are also shown for the different solvent models used: CG-FE and CG-ST.

\begin{tabular}{|c|c|c|c|c|c|c|}
\hline Ref./Sol. & System & $\begin{array}{c}\mathrm{VdW} \\
(\mathrm{kJ} / \mathrm{mol})\end{array}$ & $\begin{array}{c}\text { Elec. } \\
(\mathrm{kJ} / \mathrm{mol})\end{array}$ & $\begin{array}{c}\text { Total } \\
(\mathrm{kJ} / \mathrm{mol})\end{array}$ & $\begin{array}{c}\epsilon \\
(\mathrm{kJ} / \mathrm{mol})\end{array}$ & $\begin{array}{c}\sigma \\
(\mathrm{nm})\end{array}$ \\
\hline \multirow[t]{2}{*}{ P/W } & AA & 9.08 & -9.19 & -0.11 & - & - \\
\hline & CG & -0.21 & - & -0.21 & 2.81 & 0.288 \\
\hline \multirow[t]{2}{*}{$\mathbf{P} / \mathbf{C}$} & AA & -23.36 & - & -23.36 & - & - \\
\hline & $\mathrm{CG}$ & -23.21 & - & -23.21 & 4.515 & 0.547 \\
\hline mod-P-C CG-FE & $\mathrm{CG}$ & & - & -54.30 & 7.00 & 0.547 \\
\hline mod-P-C CG-ST & $\mathrm{CG}$ & & - & -72.59 & 8.40 & 0.547 \\
\hline \multirow[t]{2}{*}{$\mathbf{B} / \mathbf{W}^{\mathrm{a}}$} & AA & 28.81 & -283.6 & -254.8 & - & - \\
\hline & $\mathrm{CG}$ & -119.0 & - & -119.0 & 10.31 & 0.288 \\
\hline \multirow[t]{2}{*}{$\mathrm{B} / \mathrm{C}$} & AA & -18.62 & - & -18.62 & - & - \\
\hline & $\mathrm{CG}$ & -9.78 & - & -9.78 & 4.0 & 0.547 \\
\hline
\end{tabular}

${ }^{\mathrm{a}}$ For the $\mathrm{B} / \mathrm{W}$ interaction $\mathrm{CG}$ bead is tuned to reproduce half of the hydration free energy of zwitterionic diglycine. 


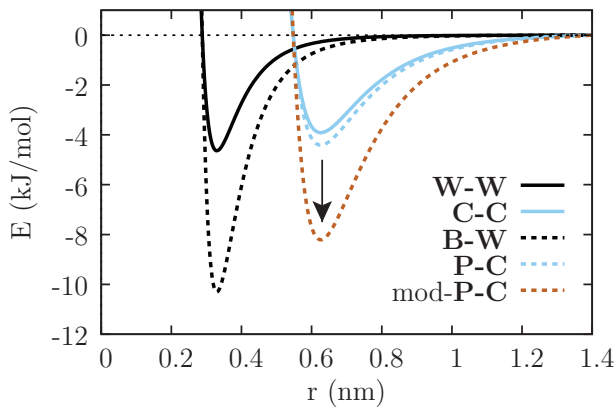

FIG. 8. CG nonbonded interaction potentials between the FF and solvent beads (Lennard-Jones parameters are given in Table II). For the P-C potential the modified CG interaction is also shown for CG-ST solvent model (dashed brown line), which corrects the dihedral distribution at the interface (see text for details).

is used in atomistic free energy calculations to parametrize the CG $\mathbf{P}$ beads. For the $\mathbf{B}$ beads, the solvation free energy of zwitterionic diglycine is computed since a smaller charge-neutral atomistic reference would have been difficult to construct. Half of its transfer free energy is used for the initial parameterization of the CG model. Peptide-peptide B-B and B-P interactions are not included, since we are only interested in a single FF molecule. The P-P interaction is set as a purely repulsive Lennard-Jones potential (short ranged interaction with a cut-off at the minimum), representing only the excluded volume effect in the CG simulations. The nonbonded interaction potentials which play an important role in the FF molecule's conformational behavior are shown in Fig. 8.

\section{Parametrization of the dihedral potential}

In this section, the parametrization of the CG dihedral potential PBBP and the performance of the FF CG model in the CG-FE and CG-ST solvent models will be discussed. We start by analyzing the behavior of the CG model in bulk water in the absence of an explicit dihedral potential. In bulk water, both CG solvent models are identical since the $\mathbf{W}-\mathbf{C}$ interactions are not relevant.

The first problem arises from the spherical bead representation of the phenylalanine side chain, which has a planar geometry. The single-bead representation of $\mathbf{P}$ prevents penetration of $\mathrm{CG}$ water beads into the space between the two sidechains, and as a result, unlike the AA bulk water simulation, the CG simulation yields a cis-like conformation (Fig. 9, dashed brown line). This can be overcome if the diameter of the $\mathbf{P}-\mathbf{W}$ interaction is reduced from (initial) $0.5 \mathrm{~nm}$ to $0.28 \mathrm{~nm}$ which corresponds to the $\sigma$ of the $\mathbf{W}-\mathbf{W}$ interaction. With these "reduced size" $\mathbf{P}$ beads, the CG model yields a uniform dihedral angle probability distribution (Fig. 9, solid brown line). In other words, with all other bonded and nonbonded interactions defined, this CG FF model has no dihedral preference on its own in bulk water. Therefore, to capture the atomistic behavior in bulk water, one can simply Boltzmann invert the atomistic dihedral probability distribution in bulk water to obtain an effective dihedral potential. After adding this dihedral potential, the CG model

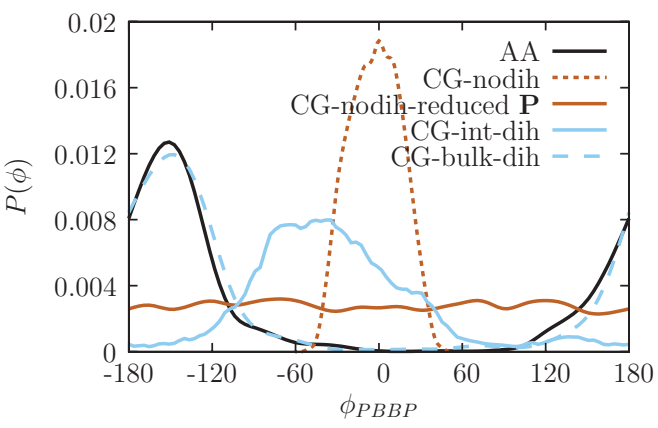

FIG. 9. Bulk water: Comparison of PBBP dihedral angle probability distribution from CG simulations with the atomistic reference (solid-black line). Without an explicit dihedral potential, single-bead representation (see text) of the $\mathbf{P}$ beads leads to a cis-like conformation (dashed brown line). When the size of the $\mathbf{P}$ beads is reduced, a flat dihedral distribution is obtained (solid brown line). Thus, dihedral potential obtained via Boltzmann Inversion (BI) of the atomistic reference in bulk water (dashed blue line) reproduces the correct behavior. On the other hand, BI of the interface distribution fails in bulk water (solid-blue line).

is perfectly capable of representing the bulk water behavior, as shown in Fig. 9 (dashed blue line). Alternatively, if one obtains a dihedral potential by Boltzmann inverting the AA simulation's dihedral distribution at the interface, a cis-like conformation is observed also in bulk water as expected (solid blue line in Fig. 9), i.e., not the appropriate bulk behavior.

Next, we use this CG model which reproduces the bulk water behavior (with reduced size $\mathbf{P}-\mathbf{W}$ interaction and dihedral potential obtained from BI of the bulk water distribution) for the biphasic system with a cyclohexane/water interface. Figure 10 shows that in the CG-ST solvent model the dihedral distribution at the interface is not correct: the FF molecule remains in a trans state (Fig. 10, solid brown line). In the CG-FE solvent model (Fig. 10, solid blue line), however, the FF molecule undergoes a transition towards a cis-like state, with a dihedral distribution that is shifted compared to the AA result, but that nevertheless at least qualitatively displays the

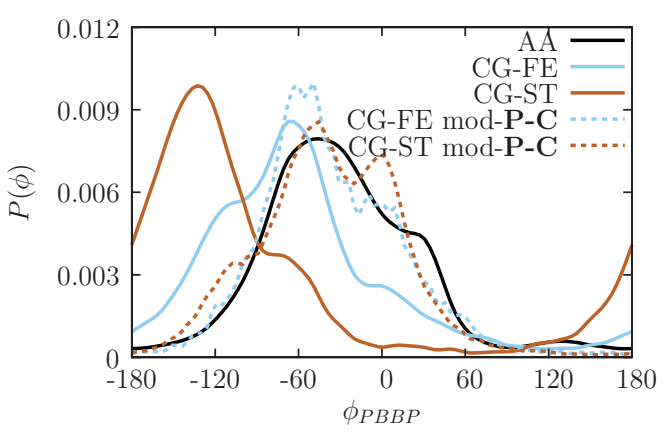

FIG. 10. Cyclohexane/water interface: Comparison of PBBP dihedral angle probability distribution from CG simulations with the atomistic reference (solid black line). CG model uses reduced $\mathbf{P}-\mathbf{W}$ interaction radius and dihedral potential based on BI of atomistic bulk water dihedral distribution. The CG solvent model with surface tension tuned $\mathbf{W}-\mathbf{C}$ interaction fails to capture the correct dihedral behavior (CG-ST, solid brown line), whereas the W-C interaction tuned to reproduce solvation free energy of a water molecule in cyclohexane solvent (CG-FE, solid blue line) yields a fairly close result. By increasing the attraction for the P-C interaction both CG models can be enhanced to yield a better match with the AA result (dashed blue and dashed brown lines for CG-FE and CG-ST solvent models, respectively). 


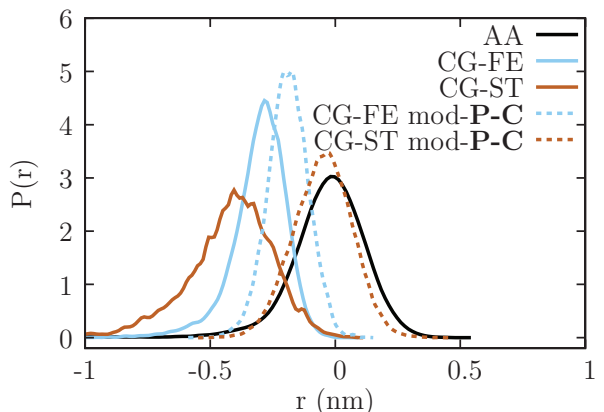

FIG. 11. Probability distribution for the distance of FF from the interface. Unlike the AA simulation (solid black line), where the molecule is placed right at the interface, CG model yields a submerged FF molecule with both of the tested solvent models (CG-FE and CG-ST shown with solid blue and solid brown lines, respectively), with CG-ST model displaying looser adsorption to the interface. Modified P-C interaction enhances both cases (dashed lines), where modified CG-ST case yields almost a perfect match with atomistic results.

correct conformational transition. This is in a sense quite a remarkable difference since the two solvent models only differ in the interaction between cyclohexane and water, i.e., the interface properties, there is no difference in the direct interaction between the peptide and the solvents. Nevertheless, one of the two models is showing - qualitatively correctly-the environment-driven conformational transition, and one is not. Remarkably, the model that does show the transition is not the model that had been parametrized according to the surface tension, i.e., the interface behavior. In order to understand this better, we have proceeded to analyze the behavior of FF at the interface further, and to investigate what would have to be done to reproduce the conformational change at the interface in both solvents.

As far as the positioning of the FF molecule at the interface is concerned, in both CG solvent models the FF molecule is submerged somewhat towards the water side of the interface compared to the AA reference, with the CG-FE model being closer to the AA result (see Fig. 11, solid blue and brown lines). For the orientation of the molecule with respect to the interface, the CG model does not display the broken symmetry observed in the atomistic simulation. The distribution of the orientation angle $\gamma$ has a maximum at $\gamma=90^{\circ}$ in the CG model (see Fig. 12). Here, $\gamma$ denotes the angle between the

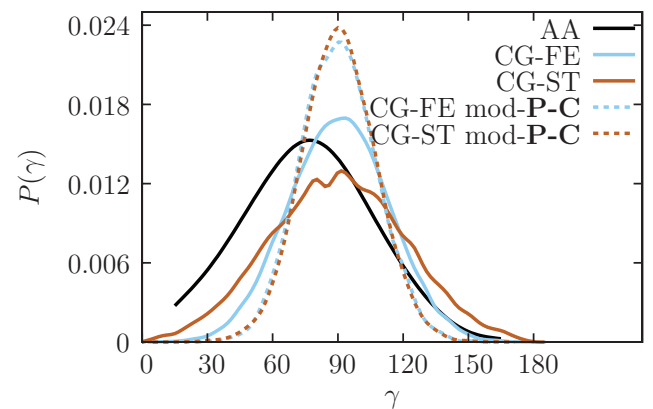

FIG. 12. Probability distribution for the orientation angle $(\gamma)$ of the molecule with respect to the interface for atomistic (solid black line) and CG model with two different solvent models (solid blue line for CG-FE and solid brown line for CG-ST). Upon modification of the P-C interaction, both CG-FE and CG-ST cases yield a narrower distribution compared to the atomistic results (dashed lines).

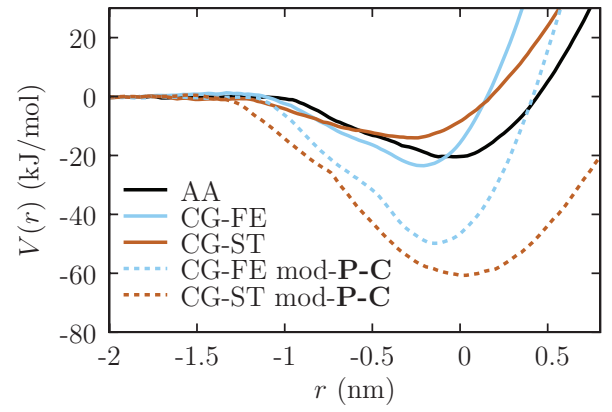

FIG. 13. Potential of mean force, $V(r)$, for the transfer of FF from bulk water to the cyclohexane/water interface. CG-FE solvent model yields the closest match to the atomistic result in terms of depth (solid blue line), whereas CGST solvent model displays a weaker attraction towards the interface. In both cases the minimum is shifted towards the water side of the interface. Modification of the P-C interaction pulls down the PMF curve to much deeper values, largely overestimating the adsorption energy (dashed lines).

interface normal and the normal of a plane fitted to the four beads of the CG peptide. With the lower resolution of the $\mathrm{CG}$ model, failure of the model to capture this broken symmetry is not surprising.

Since we assumed that the conformational transition of the molecule is driven by the segregation forces that are only present at the interface, we calculated the potential of mean force for pulling the FF molecule from the middle of the water layer to the interface. The PMF curves corresponding to the AA and CG system are shown in Fig. 13. Since we are mainly interested in the transfer from the bulk water phase to the interface, we limited our PMF calculations to a distance range up to $0.8 \mathrm{~nm}$ across the interface. The atomistic system shows a $20.6 \mathrm{~kJ} / \mathrm{mol}$ change in free energy upon adsorption of the molecule to the interface. The minimum of the potential is positioned right at the interface, in agreement with the free simulation results shown in Fig. 11. The CGFE model (Fig. 13, solid blue line) yields a similar adsorption free energy $(23.6 \mathrm{~kJ} / \mathrm{mol})$. However, unlike the atomistic system, the potential minimum is located slightly below the interface (also in agreement with the results shown in Fig. 11). The CG-ST solvent model shows a weaker adsorption to the interface (solid brown line), i.e., in this aspect it shows less agreement with the atomistic reference (solid brown line).

An interesting difference between the CG-FE and CG-ST results is that the CG-FE PMF curve rises more steeply compared to the AA PMF when the molecule is dragged into the cyclohexane phase. The analysis of the trajectory shows that this is a direct consequence of the hydration shell of the FF molecule. For the CG-FE solvent model, the reduced W-C interaction potential results in a stripping off of all the water molecules in the hydration shell of FF as the molecule is pulled out of the water layer. In contrast, the CG-ST successfully mimics the AA results in this regard and maintains part of the hydration shell of the FF molecule as the molecule enters the cyclohexane phase.

The results shown so far suggest that tuning the dihedral potential alone is not necessarily sufficient to capture the desired conformational behavior for the CG system in both bulk water and at the cyclohexane/water interface. Even though 
the affinity towards the interface (i.e., the PMF of the entire molecule) is reproduced fairly nicely with the fragmentbased free-energy-tuned peptide-solvent interactions, the cis-trans conformational transition is not necessarily correctly represented by the CG model. Disconcertingly, in the present system the outcome appears to be depending on the solventsolvent interactions, i.e., on parameters that do not involve the peptide at all and that may in some more complex scenarios of many-component systems not be free for parameter changes.

Therefore, we have investigated what would have to be done (involving the peptide-solvent interactions) to reproduce the correct conformational behavior in both solvent scenarios. We found that one possible solution involves increasing the strength of the interaction between the cyclohexane and phenylalanine side chain beads (see Fig. 8, dashed brown line, labeled mod-P-C), so that the segregation forces are further enhanced. Since the P-C interaction is used only in the interface simulation, the bulk water behavior of the molecule remains unaffected. For each of the two CG solvent models the depth of the modified P-C (listed in Table II) is chosen such that at the interface the AA cis-dihedral distribution is more or less exactly reproduced-overruling the dihedral potential which favors a trans-like conformation. Interestingly, for both CG solvent models the interaction has to be substantially deepened to achieve the effect, even though in the CG-FE case the unmodified model had already shown a qualitatively quite acceptable distribution. As shown in Fig. 10 with dashed lines, after this modification the CG model correctly captures the interface dihedral distribution with both CG solvent models. The position with respect to the interface is also improved compared to the original CG model (Fig. 11, dashed brown line), surprisingly CG-ST model now outperforming the CG-FE model. The orientation of the molecule still yields a maximum at $\gamma=90^{\circ}$ (Fig. 12) and is much narrower compared to the AA result. However, this improvement takes place at the cost of the adsorption free energy: the modified CG model (by construction-due to the stronger P-C affinity) exhibits a much larger driving force towards the interface (Fig. 13).

\section{CONCLUSIONS}

The results presented in this paper show that with respect to sampling of conformations, transferability of CG models cannot be taken for granted. The model system, diphenylalanine, displays distinctly different conformations in bulk water compared to the cyclohexane/water interface. The pseudo dihedral angle, which we labeled as PBBP favors a trans-like conformation in bulk water, whereas it switches to cis-like conformation at the cyclohexane/water interface. The same conformational transition is also seen upon aggregation of FF molecules into nano-tubes. Hence, if one desires to capture the surface adsorption or aggregation of such a molecule via a CG model, the model should display an identical conformational transition.

Here, we showed that such a conformational cis/trans transition is not automatically captured by the CG model, even if the partitioning forces that are the origin of this conformational transition form the basis of the parametrization of nonbonded interactions. Even more so, the ability to reproduce the conformational transition may depend on parameters of the solvent models that do not involve the peptide at all and that merely affect the behavior of the interface. Between the two different solvent models we have tested, the CG-ST solvent model, where the mixed solvent-solvent interactions had been parametrized according to surface tension, did not induce the conformational transition at the interface at all, even though the model showed quite good results for the surface affinity (see PMF curve). The CG-FE model on the other hand, where the water cyclohexane interaction is parameterized to mimic the solvation free energy of water in cyclohexane, yields a fairly good result in capturing the dihedral distribution at the interface and representing the adsorption free energy from water to the cyclohexane/water interface.

Finally, we showed that the interface dihedral distribution for both of these CG solvent models can be corrected by reparametrizing the $\mathbf{P}-\mathbf{C}$ interaction. This modification not only affects the conformational behavior, it also significantly improves the position of the FF molecule at the interface. However, one sacrifices the correct adsorption strength of the FF to the interface, as seen in the PMF curves in Fig. 13. The crucial role that the correct representation of the water interface has on the conformational transitions of the peptide suggests that, it will be eminently interesting to study conformational transferability in a water model such as the one from Molinero and Moore ${ }^{66}$ in the future. Improved representation of structural and thermodynamic properties of water could potentially eliminate the need for reparametrization of peptide-solvent interactions.

The entire study shows that one has to reconsider the separation of bonded and nonbonded interactions for the parametrization of CG models. The subtle interplay between the conformational preference of the molecule (that should be mainly "covered" by the dihedral angle potential), the solvation properties of its fragments (that should be mainly "covered" by the various nonbonded interaction potentials), and even interactions in the environment that do not directly affect the solute molecule at all have to be taken into account. In the present paper, we have shown that one way to correctly capture the conformational preference is to adjust the nonbonded interactions, rather than playing with the dihedral potential. This modification corrects the conformational preference of the molecule at the interface and reproduces the conformational transition, but one sacrifices the exact reproduction of the adsorption free energy to the interface.

The parametrization presented here was intended mainly to understand the interplay of the relevant driving forces for such an environmentally-driven conformational transition and how they can be correctly accounted for in the CG model. We are aware that the four-bead CG model used in this paper may be problematic for reproducing the full aggregation behavior including the formation of microscopically accurate structures, since the backbone is likely to be too coarsely represented and sidechains might fail to capture the aromatic nature of the phenylalanine sidechains. Representation of sidechain conformations could potentially be improved by using an angle dependent potential. ${ }^{67}$ Alternatively, a similar 
parametrization can be introduced for the MARTINI representation of FF molecule, which has been recently shown to exhibit interesting aggregation behavior for $\mathrm{FF}^{68}$ In the long run it would also be interesting to use a solvent free model to study large scale aggregation (without an interface). In that case the crucial interactions that will have to be balanced are the hydrophilic and hydrophobic interactions between the various peptide beads.

\section{ACKNOWLEDGMENTS}

M. Sayar, C. Dalgicdir, and O. Sensoy would like to thank TUBITAK (Grant No. 212T184). We also thank the Max Planck Society for financial support through the Partner Group Agreement with Professor Kurt Kremer's Theory Group at MPIP, Mainz. C. Peter gratefully acknowledges financial support by the German Science Foundation within the Emmy Noether Programme (Grant No. PE 1625/1). We are grateful to Christoph Globisch and Alok Jain for many valuable suggestions.

${ }^{1}$ M. Gustafsson, G. Vandenbussche, T. Curstedt, J. Ruysschaert, and J. Johansson, FEBS Lett. 384(2), 185 (1996).

${ }^{2}$ W. DeGrado, H. Gratkowski, and J. Lear, Protein Sci. 12(4), 647 (2003).

${ }^{3}$ M. B. Ulmschneider, J. P. F. Doux, J. A. Killian, J. C. Smith, and J. P. Ulmschneider, J. Am. Chem. Soc. 132(10), 3452 (2010).

${ }^{4}$ A. L. Boyle and D. N. Woolfson, Chem. Soc. Rev. 40(8), 4295 (2011).

${ }^{5}$ C. M. Dobson, Nature (London) 426(6968), 884 (2003).

${ }^{6}$ F. Chiti and C. M. Dobson, Annu. Rev. Biochem. 75, 333 (2006).

${ }^{7}$ C. Wu and J.-E. Shea, Curr. Opin. Struct. Biol. 21, 209 (2011).

${ }^{8}$ C. Schladitz, E. P. Vieira, H. Hermel, and H. Möhwald, Biophys. J. 77(6), 3305 (1999).

${ }^{9}$ H. Rapaport, Supramol. Chem. 18(5), 445 (2006).

${ }^{10}$ R. Fairman and K. S. Akerfeldt, Curr. Opin. Struct. Biol. 15(4), 453 (2005).

${ }^{11}$ K. Rajagopal and J. P. Schneider, Curr. Opin. Struct. Biol. 14(4), 480 (2004).

${ }^{12}$ H. Rapaport, H. Grisaru, and T. Silberstein, Adv. Funct. Mater. 18(19), 2889 (2008).

${ }^{13}$ W. Li, F. Nicol, and F. Szoka, Adv. Drug Delivery Rev. 56(7), 967 (2004).

${ }^{14}$ M. C. Branco, D. M. Sigano, and J. P. Schneider, Curr. Opin. Chem. Biol. 15(3), 427 (2011).

${ }^{15}$ C. H. Görbitz, Chem.-Eur. J. 7(23), 5153 (2001).

${ }^{16}$ M. Reches, Science 300(5619), 625 (2003).

${ }^{17}$ S. Pronk, S. Páll, R. Schulz, P. Larsson, P. Bjelkmar, R. Apostolov, M. R. Shirts, J. C. Smith, P. M. Kasson, D. van der Spoel et al., Bioinformatics 29(7), 845 (2013).

${ }^{18}$ C. Oostenbrink, A. Villa, A. E. Mark, and W. F. van Gunsteren, J. Comput. Chem. 25(13), 1656 (2004).

${ }^{19}$ H. J. C. Berendsen, J. R. Grigera, and T. P. Straatsma, J. Phys. Chem. 91(24), 6269 (1987).

${ }^{20}$ B. Hess, H. Bekker, H. J. C. Berendsen, and J. G. E. M. Fraaije, J. Comput. Chem. 18(12), 1463 (1997).

${ }^{21}$ G. Bussi, D. Donadio, and M. Parrinello, J. Chem. Phys. 126(1), 014101 (2007).

${ }^{22}$ U. Essmann, L. Perera, and M. T. Berkowitz, J. Chem. Phys. 103(19), 8577 (1995).

${ }^{23}$ V. Rühle and C. Junghans, Macromol. Theory Simul. 20(7), 472 (2011).

${ }^{24}$ R. Apostolov, H. Berendsen, A. Van Buuren, P. Bjelkmar, R. Van Drunen, A. Feenstra, G. Groenhof, P. Kasson, P. Larsson et al., Gromacs Manual 4.5 (Royal Institute of Technology and Uppsala University, Stockholm and Uppsala, Sweden, 2010).

${ }^{25}$ W. Humphrey, A. Dalke, and K. Schulten, J. Mol. Graphics 14(1), 33 (1996).
${ }^{26}$ See supplementary material at http://dx.doi.org/10.1063/1.4848675 for simulation details.

${ }^{27}$ C. H. Bennett, J. Comput. Phys. 22, 245 (1976).

${ }^{28}$ D. Wu and D. A. Kofke, J. Chem. Phys. 123(8), 084109 (2005).

${ }^{29}$ W. F. van Gunsteren and H. J. C. Berendsen, Mol. Simul. 1(3), 173 (1988).

${ }^{30}$ H. J. C. Berendsen, J. P. M. Postma, and W. F. van Gunsteren, J. Chem. Phys. 81(8), 3684 (1984).

${ }^{31}$ G. M. Torrie and J. P. Valleau, Chem. Phys. Lett. 28(4), 578 (1974).

${ }^{32}$ O. Engin, A. Villa, M. Sayar, and B. Hess, J. Phys. Chem. B 114(34), 11093 (2010).

${ }^{33}$ S. Kumar, J. M. Rosenberg, D. Bouzida, R. H. Swendsen, and P. A. Kollman, J. Comput. Chem. 13(8), 1011 (1992).

${ }^{34}$ V. Tozzini, Q. Rev. Biophys. 43, 333 (2010).

${ }^{35}$ O. Bezkorovaynaya, A. Lukyanov, K. Kremer, and C. Peter, J. Comput. Chem. 33, 937 (2012).

${ }^{36}$ W. Tschöp, K. Kremer, J. Batoulis, T. Burger, and O. Hahn, Acta Polym. 49(2-3), 61 (1998).

${ }^{37}$ C. Peter and K. Kremer, Soft Matter 5, 4357 (2009).

${ }^{38}$ O. Engin, A. Villa, C. Peter, and M. Sayar, Macromol. Theory Simul. 20, 451 (2011).

${ }^{39}$ S. O. Nielsen, C. F. Lopez, G. Srinivas, and M. L. Klein, J. Chem. Phys. 119, 7043 (2003).

${ }^{40}$ S. J. Marrink, A. H. deVries, and A. E. Mark, J. Phys. Chem. B 108, 750 (2004).

${ }^{41}$ S. J. Marrink, H. J. Risselada, S. Yefimov, D. P. Tieleman, and A. H. de Vries, J. Phys. Chem. B 111, 7812 (2007).

${ }^{42}$ B. M. Mognetti, L. Yelash, P. Virnau, W. Paul, K. Binder, M. Mueller, and L. G. Macdowell, J. Chem. Phys. 128, 104501 (2008).

${ }^{43}$ R. DeVane, W. Shinoda, P. B. Moore, and M. L. Klein, J. Chem. Theory Comput. 5, 2115 (2009).

${ }^{44}$ A. P. Lyubartsev and A. Laaksonen, Phys. Rev. E 52, 3730 (1995).

${ }^{45}$ F. Müller-Plathe, ChemPhysChem 3, 754 (2002).

${ }^{46}$ D. Reith, M. Putz, and F. Müller-Plathe, J. Comput. Chem. 24, 1624 (2003).

${ }^{47}$ C. Peter, L. Delle Site, and K. Kremer, Soft Matter 4, 859 (2008).

${ }^{48}$ T. Murtola, M. Karttunen, and I. Vattulainen, J. Chem. Phys. 131, 055101 (2009).

${ }^{49}$ A. Lyubartsev, A. Mirzoev, L. J. Chen, and A. Laaksonen, Faraday Discuss. 144, 43 (2010).

${ }^{50}$ A. Savelyev and G. A. Papoian, J. Phys. Chem. B 113, 7785 (2009).

${ }^{51}$ G. Megariotis, A. Vyrkou, A. Leygue, and D. N. Theodorou, Ind. Eng. Chem. Res. 50, 546 (2011).

${ }^{52}$ B. Mukherjee, L. D. Site, K. Kremer, and C. Peter, J. Phys. Chem. B 116(29), 8474 (2012).

${ }^{53}$ S. Izvekov and G. A. Voth, J. Phys. Chem. B 109, 2469 (2005).

${ }^{54}$ W. G. Noid, J.-W. Chu, G. S. Ayton, V. Krishna, S. Izvekov, G. A. Voth, A. Das, and H. C. Andersen, J. Chem. Phys. 128, 244114 (2008).

${ }^{55}$ M. S. Shell, J. Chem. Phys. 129, 144108 (2008).

${ }^{56}$ M. E. Johnson, T. Head-Gordon, and A. A. Louis, J. Chem. Phys. 126, 144509 (2007).

${ }^{57}$ J. R. Silbermann, S. H. L. Klapp, M. Schoen, N. Chennamsetty, H. Bock, and K. E. Gubbins, J. Chem. Phys. 124, 074105 (2006).

${ }^{58}$ E. C. Allen and G. C. Rutledge, J. Chem. Phys. 130, 034904 (2009).

${ }^{59}$ J. W. Mullinax and W. G. Noid, J. Chem. Phys. 131, 104110 (2009).

${ }^{60}$ A. Villa, C. Peter, and N. F. A. van der Vegt, J. Chem. Theory Comput. 6, 2434 (2010).

${ }^{61}$ S. Izvekov, P. W. Chung, and B. M. Rice, J. Chem. Phys. 133, 064109 (2010).

${ }^{62}$ J.-W. Shen, C. Li, N. F. A. van der Vegt, and C. Peter, J. Chem. Theory Comput. 7, 1916 (2011).

${ }^{63}$ E. Brini, V. Marcon, and N. F. A. van der Vegt, Phys. Chem. Chem. Phys. 13, 10468 (2011).

${ }^{64}$ J. Jeon, C. E. Mills, and M. S. Shell, J. Phys. Chem. B 117(15), 3935 (2013).

${ }^{65}$ J. G. Kirkwood, J. Chem. Phys. 3(5), 300 (1935).

${ }^{66}$ V. Molinero and E. B. Moore, J. Phys. Chem. B 113(13), 4008 (2009).

${ }^{67}$ N. V. Buchete, J. E. Straub, and D. Thirumalai, Curr. Opin. Struct. Biol. 14(2), 225 (2004).

${ }^{68}$ C. Guo, Y. Luo, R. Zhou, and G. Wei, ACS Nano 6(5), 3907 (2012). 